Published in final edited form as:

Am J Sports Med. 2019 July ; 47(8): 1831-1843. doi:10.1177/0363546519850572.

\title{
Changes in Cross-Sectional Area and Signal Intensity of Healing ACLs and ACL Grafts in the First Two Years After Surgery
}

\author{
Ata M. Kiapour, PhD ${ }^{1}$, Kirsten Ecklund, MD², Martha M. Murray, MD $^{1}$, BEAR Trial Team, and \\ Braden C. Fleming, PhD $^{3,4}$ \\ ${ }^{1}$ Department of Orthopaedic Surgery, Boston Children's Hospital, Harvard Medical School, \\ Boston MA 02115 \\ ${ }^{2}$ Department of Radiology, Boston Children's Hospital, Harvard Medical School, Boston MA \\ 02115
}

${ }^{3}$ Department of Orthopaedics, Warren Alpert Medical School of Brown University/Rhode Island Hospital, Providence, RI 02818

${ }^{4}$ School of Engineering, Brown University, Providence, RI 02818

\begin{abstract}
Background: The quality of a repaired anterior cruciate ligament (ACL) or reconstructed graft is typically quantified in clinical studies by evaluating knee, lower extremity or subject performance. However, magnetic resonance (MR) imaging of the healing ACL or graft may provide a more direct measure of the tissue quality (i.e., signal intensity) and quantity (i.e., cross-sectional area).

Hypothesis: 1) Average cross-sectional area or signal intensity of a healing ACL following Bridge-Enhanced ACL Repair (BEAR) of a midsubstance ACL tear or a hamstring autograft (ACLR) will change postoperatively from 3 to 24 months. 2) The average cross-sectional area and signal intensity of the healing ligament or graft will correlate with anatomical features of the knee associated with ACL injury.
\end{abstract}

Study Design: Cohort Study; Level of evidence, 2.

Methods: Subjects with a complete, midsubstance ACL tear who were treated with either BEAR $(\mathrm{n}=10)$ or $\operatorname{ACLR}(\mathrm{n}=10)$ underwent MR imaging at 3, 6, $12 \& 24$ months after surgery. Images were analyzed to determine the average cross-sectional area and signal intensity of the ACL or graft at each time point. ACL orientation, stump length and bony anatomy were also assessed.

Corresponding Author: Ata M. Kiapour, PhD MMSc, Orthopaedic Research Laboratories, Departmentof Orthopaedic Surgery, Boston Children's Hospital, Boston, Harvard Medical School, 300 Longwood Avenue 02115, Phone: (617) 919-2033, ata.kiapour@childrens.harvard.edu.

Disclosures: MMM and Boston Children's Hospital have equity interests in MIACH Orthopaedics, a Company that has licensed the BEAR scaffolding technology from Boston Children's Hospital. MMM also receives royalties from Springer Publishing and research grants from the NIH, DoD and NFLPA through the Harvard Football Players Health Study. BCF is a co-founder of MIACH Orthopaedics, is an associate editor for the American Journal of Sports Medicine and receives royalties from Springer Publishing.

The BEAR Trial Team: Brett Flutie, Christina Freiberger, Rachael Henderson, Dennis Kramer, Lyle Micheli, Laura Thurber and YiMeng Yen. All are affiliated with the Division of Sports Medicine, Department of Orthopaedic Surgery, Boston Children's Hospital Boston, MA 02115.

The investigation was performed at Boston Children's Hospital, Boston, MA 
Results: Mean cross-sectional area of the grafts was 48-98\% larger than the contralateral intact ACL at all time points ( $\mathrm{p}<.01)$. The BEAR ACLs were $23-28 \%$ greater than the contralateral intact ACL at 3 and 6 months ( $\mathrm{p}<.02)$, but similar at 12 and 24 months. The BEAR ACLs were similar in sagittal orientation to the contralateral ACL while the grafts were $6.5^{\circ}$ more vertical $(\mathrm{p}=$. 005). For the BEAR ACLs, the bigger notch correlated with bigger cross-sectional area, while a shorter ACL femoral stump, steeper lateral tibial slope and shallower medial tibial depth were associated with higher signal intensity $\left(\mathrm{R}^{2}>.40 ; \mathrm{p}<.05\right)$. Performance of notchplasty resulted in increased ACL cross-sectional area after the BEAR procedure ( $\mathrm{p}=.007)$. No anatomic features were correlated with ACL graft size or signal intensity.

Conclusions: Hamstring autografts were larger in cross-sectional area and more vertically oriented than the native ACL at 24 months after surgery. BEAR ACLs have a cross-sectional area, signal intensity and sagittal orientation that were similar to the contralateral ACL at 24 months. The early signal intensity and cross-sectional area of the repaired ACL may be affected by specific anatomic features, including lateral tibial slope and notch width, observations that deserve further study in a larger cohort of subjects.

\section{Social Media Summary:}

A new study shows the changes in ligament structure within 2 years after Bridge-Enhanced ACL Repair (BEAR) and ACL reconstruction. Repaired ACLs had a cross-sectional area, signal intensity and sagittal orientation that were similar to the contralateral ACL at 24 months.

\section{Keywords}

ACL; Repair; Bridge-enhanced ACL Repair; BEAR; Reconstruction; MRI; Signal Intensity; Size

\section{INTRODUCTION}

Currently, the integrity of an anterior cruciate ligament (ACL) graft or repair is quantified in clinical studies by evaluating whole knee function, lower extremity performance and/or patient-reported outcomes. However, these measures are often influenced by factors unrelated to the ACL structure. For example, physical examinations of the knee, e.g., the Lachman and pivot shift tests, can be influenced by the injury or hypertrophy of secondary stabilizers of the knee ${ }^{17,55}$ as well as patient age, ${ }^{33}$ sex, ${ }^{33}$ bony anatomy, ${ }^{41}$ and are prone to observer bias. Functional testing, for example hop testing and balance testing, can be influenced by the quality of the rehabilitation program, patient compliance and/or fear of reinjury. ${ }^{10,46}$ Likewise, patient-reported outcomes after ACL surgery have been shown to be influenced by self-esteem levels, ${ }^{11}$ body mass index, ${ }^{18}$ and smoking. ${ }^{18}$ When evaluating the success or failure of a new surgical procedure, clinical, functional and patient-reported outcomes may be less sensitive to evaluate the structural integrity of a healing ACL or ACL graft due to these non-structural related factors. Direct measures of graft or ligament quality, including the size and degree of tissue organization, could potentially improve our ability to compare the ability of different surgical procedures to restore the ACL structure.

Magnetic resonance (MR) imaging has been previously used in preclinical ${ }^{6,7}$ and clinical $^{5,28-30}$ studies evaluating ACL graft quality and associated outcomes. In a large 
animal study, it was shown that the combination of ACL graft size (a measure of tissue quantity) and signal intensity (a measure of tissue quality) determined from specific MRI sequences were associated with the graft mechanical ${ }^{6,7}$ and histological ${ }^{49}$ properties after ACL reconstruction. ACL graft signal intensity (measured from MRI in 2D) has shown to be predictive of anterior tibial translation 3 months after surgery but not clinical and functional outcomes at 1 year. ${ }^{16}$ However, a 3D assessment of ACL graft signal intensity and size have been shown to be predictive of a range of functional, patient-reported and clinical outcomes three to five years after ACL reconstruction in humans. ${ }^{5}$ These studies highlight the potential of these MR-based parameters for its noninvasive and nondestructive nature, MR imaging can be used to study changes in cross-sectional area and signal intensity of grafts and ligaments at multiple time points after surgery. This approach could be useful for the comparison of different surgical procedures, or to evaluate factors (e.g., notch width) influencing ligament healing following surgical treatment.

In this study, a quantitative MR imaging approach was used to evaluate time-dependent changes in graft size and signal intensity over the first two years after ACL reconstruction (ACLR). This approach was also used to study structural changes for complete, midsubstance ACL tears treated with a novel surgical repair procedure, Bridge-Enhanced ACL Repair (BEAR). ${ }^{38,47}$ Prior attempts at primary ACL repair for midsubstance ACL tears have had a relatively high failure rate, ${ }^{15}$ and previous basic and translational research has suggested it may be a premature loss of the provisional scaffold at the time of injury due to the location of the ACL in the synovial fluid environment. ${ }^{40}$ BEAR aims to address that deficiency by surgically placing a substitute provisional scaffold in the gap in the midsubstance of the torn ACL to stimulate healing at the time of suture repair. ${ }^{36,} 37$ A recent safety study evaluated the first ten subjects, who underwent the BEAR procedure, and demonstrated similar patient-reported outcomes and anteroposterior (AP) knee laxity compared to a control group treated with hamstring autograft ACLR at 3 month and two years follow-up. ${ }^{38,39}$

The primary objectives of this study were two-fold. First, we utilized MR images from these two cohorts to compare changes over time in terms of restoring the native ligament size (average cross-sectional area) and tissue quality (normalized signal intensity). We hypothesized that there would be differences in the average cross-sectional area or average normalized signal intensity of the repaired ACL or reconstructed graft over time (i.e., 3, 6, 12 and 24 months post-operatively). Second, we determined if the ligament or graft crosssectional area and normalized signal intensity values were influenced by other anatomical features of the knee after surgery. Posterior slope of the tibial plateau, depth of the medial tibial plateau and notch width are anatomical factors linked to increased risk of ACL injury, 20,60 graft failure ${ }^{27,53}$ and inferior ACL reconstruction outcomes. ${ }^{23}$ In addition of the biomechanical role of these anatomical features on ACL/graft loading, the length of the ACL stump may also be an influencing factor on the repaired ACL remodeling during healing. Thus, we hypothesized that the ACL stump length, posterior tibial slopes, medial tibial plateau depth, and femoral notch size were associated with the average cross-sectional area and average normalized signal intensity of the healing ACL or ACL graft. 


\section{METHODS}

\section{Trial Design}

FDA Investigational Device Exemption (G140151) and Institutional Review Board (P0012985) were obtained prior to initiating the study. The trial was registered on ClinicalTrials.gov (NCT02292004). All patients granted their informed consent prior to participating. This cohort study was designed as an interventional, parallel assignment, nonrandomized, first-in-human trial for the BEAR technique. ${ }^{38}$ Ten patients were enrolled in the interventional (BEAR) group and ten in the control (ACLR) group. Enrollment was completed from February to October of 2015. Patients were evaluated pre-operatively, intraoperatively and post-operatively at 3, 6, 12 and 24 months after surgery. A detailed description of the trial was reported previously along with the description of the clinical and functional outcomes at 3 month and two years follow-up. ${ }^{38,40}$

\section{Participants}

Patients, ages 18 to 35, with a complete, midsubstance ACL tear who were less than one month from injury were eligible for enrollment in the BEAR group. ${ }^{38}$ Patients with a complete ACL tear, who were within three months of injury, were eligible to enroll in the ACLR group, all of whom received an autograft hamstring tendon graft. ${ }^{38}$ Patients with a partial ACL tear were not eligible for participation. Patients were excluded from either group if they had a prior history of knee surgery, history of knee infection, or other risk factors that might adversely affect healing (nicotine/tobacco use, corticosteroids in the past 6 months, chemotherapy, diabetes, inflammatory arthritis). Patients were excluded if they had a displaced bucket-handle tear of the medial meniscus that required repair, however, all other meniscal injuries were included. Additionally, patients were excluded if they had a fullthickness chondral injury, a grade $3 \mathrm{MCL}$ injury, a concurrent complete patellar dislocation, or an operative posterolateral corner injury.

A total of 242 patients presenting with an ACL injury were screened for participation in this study (Figure 1). Patients were identified as possible candidates if they scheduled an appointment in our sports medicine clinic with a new knee injury and had a MR scan confirming an ACL tear or if they contacted our research coordinator after hearing about the study. Of the 242 patients screened, 22 enrolled, of which 2 were excluded before surgery: 1 due to a history of corticosteroid use not discovered at the initial enrollment meeting, and the other elected to move to Florida for school (Figure 1). The primary reason for exclusion prior to enrollment was patient age $(n=181$ patients $) .{ }^{38}$ Details of the included patients have been previously reported by Murray et al. ${ }^{38}$

\section{The BEAR Scaffold}

The BEAR scaffold was comprised of bovine extracellular matrix proteins, including collagen, and underwent extensive preclinical testing prior to FDA approval for this study. 47, 48 The scaffold measured $22 \mathrm{~mm}$ in diameter by $45 \mathrm{~mm}$ in length. The scaffold softens when blood is added to it, making it conformable to the intra-articular notch and able to fill in the irregular contours of the gap between the torn ligament ends. The efficacy of the 
scaffold for stimulating ACL healing was previously demonstrated in preclinical studies. 36,37

\section{Surgical Procedures}

Bridge-Enhanced ACL Repair: The surgical steps for the BEAR procedure are shown in Figure 2. After the induction of general anesthesia, an examination was performed to verify the positive pivot shift on the injured side and to record the Lachman test, range of motion and pivot shift exam results on both knees. A knee arthroscopy was performed and any meniscal injuries were treated if present. A tibial aimer (Acufex Director Drill Guide; Smith and Nephew) was used to place a $2.4 \mathrm{~mm}$ guide pin through the tibia and anterior to the tibial footprint of the ACL. The pin was over-drilled with a $4.5 \mathrm{~mm}$ reamer (Endoscopic Drill; Smith \& Nephew). A notchplasty was performed at the surgeon's discretion to visualize the femoral stump footprint as a landmark for placement of the femoral tunnel. Care was taken not to disturb the femoral footprint of the torn ACL during the BEAR procedure. A guide pin was placed anteriorly and inferiorly (within $2 \mathrm{~mm}$ ) of the femoral ACL footprint, drilled through the femur and then over-drilled with the $4.5 \mathrm{~mm}$ reamer. A $4 \mathrm{~cm}$ arthrotomy was made at the medial border of the patellar tendon and a whip stitch of \#2 absorbable braided suture (Vicryl; Ethicon) was placed into the tibial stump of the torn ACL and the free ends subsequently passed through the femoral tunnel. A suture cinch (BEAR-Cinch; Boston Children's Hospital) comprised of two \#2 non-absorbable braided sutures (Ethibond; Ethicon) looped through the two center holes of a cortical button (Endobutton; Smith \& Nephew) was used to reduce the abnormal AP laxity of the knee by passing the cortical button through the femur and engaging it on the lateral femoral cortex, then passing the sutures through an extracellular matrix scaffold (BEAR Scaffold; Boston Children's Hospital), and then passing the sutures through the tibial tunnel and through a second cortical button. Prior to tightening the suture cinch, $10 \mathrm{cc}$ of autologous blood was obtained from the antecubital vein and added to the scaffold. The scaffold was then passed up along the sutures into the femoral notch and the knee extended. The suture cinch was tightened to reduce the AP laxity of the knee and the sutures tied over the tibial button with maximum manual tension. The sutures from the ACL tibial stump were tightened to bring the ACL stump into the scaffold and tied over the femoral cortical button. The arthrotomy was closed in layers.

ACL reconstruction with autologous hamstring tendon (ACLR): A standard hamstring autograft procedure was performed using a quadruple semitendinosus-gracilis graft looped over a continuous-loop cortical button (Endobutton, Smith \& Nephew) for proximal fixation. A bioabsorbable interference screw (BioRCI HA, Smith \& Nephew) was used for tibial fixation. ${ }^{38} \mathrm{~A}$ minimal notchplasty was performed at the surgeon's discretion as needed for adequate visualization of the posterior notch for placement of the femoral tunnel starting point within the prior ACL footprint. The femoral tunnel was drilled using an anteromedial portal technique and a flexible drill system (Clancy Anatomic Cruciate Guide, Smith \& Nephew). 
Post-operative rehabilitation: Rehabilitation protocols were identical in both BEAR and ACLR groups, including restricted range of motion from $0^{\circ}$ to $50^{\circ}$ for two weeks, use of a cold therapy unit, standardized physical therapy and use of a functional ACL brace.

\section{MR Imaging}

MR images were acquired pre-operatively (10 BEAR, 10 ACLR) and from operated knees at 3 (10 BEAR, 10 ACLR), 6 (10 BEAR, 8 ACLR), 12 (9 BEAR, 6 ACLR) and 24 (9 BEAR, 7 ACLR) months after surgery. Using a 3T scanner (Tim Trio, Siemens, Erlangan, Germany) and a 15-channel knee coil, the following sequences were obtained: coronal proton density fast spin echo with fat suppression (PD FSE; TR/TE $=3000 / 19 \mathrm{msec}, 16 \mathrm{~cm}$ FOV, $3 \mathrm{~mm}$ slice thickness, 0.3 gap, $284 \times 384$ matrix, ETL 4 ), sagittal isotropic 3D proton density fast spin echo (SPACE: TR/TE 1000/39 msec, $16 \mathrm{~cm}$ FOV, $0.5 \mathrm{~mm}$ slice reconstruction, $320 \times 320$ matrix, ETL 74), and a 3D Constructive Interference in Steady State (CISS; TR/TE $=14 / 7 \mathrm{msec}, \mathrm{FA}=35,16 \mathrm{~cm}$ FOV, $80 \times 512 \times 512$ (slice $\mathrm{x}$ frequency $\mathrm{x}$ phase)). Images using the CISS sequence were also acquired of the contralateral knee at 24 months (7 BEAR, 7 ACLR) after surgery. 3D SPACE images were reformatted in the coronal and axial planes.

\section{Imaging Outcomes}

The following parameters were quantified from the MR image stack for both BEAR and ACLR groups:

Ligament Tissue Parameters: Repaired ACLs, reconstructed grafts and contralateral intact ACLs were manually segmented from the sagittal CISS image stack to create a 3D model of the structure using commercially available software (Mimics 17.0; Materialize). ${ }^{5}$ The model was used to measure the ligament volume and length, which were then used to calculate the mean ACL cross-sectional area (Volume/Length). This approach was performed to avoid measurement challenges associated with inconsistent selection of the axial oblique slice to quantify ACL cross sectional are in its midsubstance. The average grayscale value across the ligament voxels for each patient was also extracted and normalized to the subject-specific grayscale value of the femoral cortical bone to minimize interscan variability. ${ }^{5}, 6$ The normalized grayscale value was defined as ligament signal intensity. The segmentations were done by an experienced examiner (AMK). To assess the reliability of the measurements, a subset of 20 repaired and 18 reconstructed ligaments were also segmented by an independent examiner. ${ }^{14}$

Anatomical Parameters: All other anatomical measurements were performed using MR image viewing software (Osirix Viewer v8.5, Pixmeo SARL). These included tunnel and ligament insertion positions, sagittal elevation angle, ACL stump length, tibial slopes and depth, and the femoral notch width as described below (Supplementary Figure S2).

Tunnel and Ligament Insertion Positions: The sagittal PD FSE MR images obtained at 3 months were used to document the locations of the femoral and tibial tunnels for both BEAR and ACLR knees. ${ }^{16}$ For femoral tunnels, the first sagittal slice showing the tunnel on the lateral condyle was selected and the width and height of the lateral condyle were 
measured. The distances from the center of the tunnel to the back and bottom of the condyle were measured and normalized to the width and height of the lateral condyle to quantify the location of the femoral tunnel in anterior-posterior and superior-inferior directions. Using the same images, the most medial slice showing the tibial tunnel was used to measure the width of tibia. The distance between the tibial tunnel center and the front of the tibia was also measured and then normalized to the tibial width to quantify the tibial tunnel anteriorposterior location. Similar methods were used to quantify the location of the ACL femoral and tibial insertions using pre-operative MR images.

Ligament Sagittal Elevation Angle: ACL or graft elevation angles in the sagittal plane were measured using the 3D CISS MR scans obtained at 24 months. Briefly, the longitudinal axis of the tibia was established using the technique described by Hudek et $\mathrm{al}^{21}$ in a central sagittal slice in which the tibial attachment of the PCL, the intercondylar eminence, and the anterior and posterior tibial cortices appeared in a concave shape. Then two circles were fitted to the tibial head: a cranial circle touching the anterior, posterior and cranial tibial cortex, and a caudal circle touching the anterior and posterior tibial cortex. The line connecting the center of the two circles was defined as longitudinal tibial axis. The perpendicular line to the longitudinal axis of the tibia was then established as the reference for measuring the sagittal elevation angle of the ACL or graft. The ligament longitudinal axis was defined as the line passing through the center of the tissue parallel to the anterior and posterior edges of the ligament. The ligament sagittal elevation angle was measured as the angle between the longitudinal axis of the ligament and the reference line. This method has been used previously to measure the multi-planar orientation of human ACL graft. ${ }^{54}$

ACL Stump Length: The pre-operative sagittal PD FSE MR images of the injured knee were used to measure the ACL stump length at both femoral and tibial sides. Femoral stump length was defined as the linear distance from the center of the ACL femoral insertion to the most distal fibers of the femoral remnant. Similarly, the tibial stump length was defined as the linear distance from the center of the ACL tibial insertion to the most superior fibers of the tibial remnant. Stump lengths were then normalized to the ACL length, measured as the linear distance between the center of the femoral and tibial insertions in the same MRI scans. $^{12}$

Tibial Slopes and Depth: The posterior slopes of the medial and lateral tibial plateau were measured from the 3 month sagittal PD FSE MR scans of the injured knee. ${ }^{19,21}$ The posterior slopes of the tibial plateau were measured in a sagittal slice at the center of each medial and lateral plateaus as the angle between a line that joined the peak points on the anterior and posterior rims of the plateau and a line perpendicular to longitudinal axis of the tibia. ${ }^{19}$ The medial tibial depth was also measured as the perpendicular distance between a line connecting the anterior and posterior rims of the medial tibial plateau and the deepest point of the medial plateau in the same slice that the medial slope was measured. ${ }^{19}$

Femoral Notch Width: Notch width was measured parallel to a line along the most inferior aspects of the femoral condyles in an axial slice corresponding to the front of the notch. At each time point, notch width was measured at multiple spots from middle to the 
bottom of the notch and the maximum value was used as notch width. ${ }^{45}$ The measurements were done on pre-operative and 3 months PD FSE MR images of the injured knee.

Notchplasty was defined as the difference between the notch width measured on the postoperative MR images and that measured on the pre-operative MR images.

\section{Statistical Analysis}

Imaging outcomes were summarized with descriptive statistics. Two-sample t-tests were used to compare the baseline anatomical parameters between the ACLR and BEAR groups. Mixed linear model with Bonferroni posthoc tests were used to compare the ligament crosssectional area and signal intensity at each time point and with the contralateral intact ACL. This analysis was done to address missing data points during longitudinal follow ups. The model had random intercept and slope. Time was used as the fixed effect in the model to assess time-dependent changes in the outcomes. Paired-sample t-tests were used to compare the ACL insertion, tunnel locations and ligament sagittal elevation between pre-and postsurgery or between the surgical and the contralateral knees. Two-sided p-values are reported and considered significant when $\mathrm{p}<.05$. Univariate linear regression analyses were performed to assess the associations between anatomical predictors and cross-sectional area or signal intensity of the repaired ACLs or reconstructed grafts at 24 months. Analyses were performed using statistical software (Prism v7.0, GraphPad Software Inc).

\section{RESULTS}

The baseline characteristics of both groups have been previously reported. ${ }^{38}$ In summary, the 2 groups were similar with regard to age, sex, race, and body mass index (BMI). The mean age of the patients was 24 years in both groups. The majority of the injuries in both groups were noncontact and occurred during sports participation. The normalized tibial stump length (tibial stump length/total ACL length) as measured on MRI was $49.4 \pm 11.4 \%$ (range $31 \%$ to $64 \%$ ) for the BEAR group and $53.9 \pm 11.4 \%$, (range $31 \%$ to $68 \%$ ) in the ACLR group. The quadruple hamstring tendon grafts measured either 8 or $9 \mathrm{~mm}$ in diameter at surgery. The anatomical parameters for both the ACLR and BEAR groups were also similar in terms of posterior tibial slope, medial tibial depth, and pre-operative notch size (Table 1).

\section{Time-Dependent Changes in Cross-Sectional Area}

The cross-sectional area measurements from the MR images were highly reproducible (ICC=0.959). The time-dependent changes in ACL or graft cross-sectional area are shown in Figure 3A. The ACL/graft cross-sectional area underwent significant changes within the first two years in both groups (BEAR: $\mathrm{F}=6.0, \mathrm{df}=31.8, \mathrm{P}=.001$; ACLR: $\mathrm{F}=28.4, \mathrm{df}=22.1, \mathrm{P}<.001$ ). At 3 months, the grafts had approximately double the cross-sectional area of the contralateral intact ACL ( $\mathrm{p}<.001)$. Between months 3 and 24, the ACL grafts decreased in cross-sectional area; however, they remained 48\% larger than the native ACL at 24 months postoperatively $(\mathrm{p}=.002)$. The ACLs treated with BEAR were $28 \%$ larger than the contralateral intact ACL at the 3-month time point $(\mathrm{p}=.003)$. While repaired ACL's crosssectional area remained significantly larger than the contralateral intact ACL at 3 and 6 months ( $\mathrm{p}=.019$ ), it became similar in size to the contralateral ACL at 12 and 24 months after surgery $(\mathrm{p}>.9)$. 


\section{Time-Dependent Changes in Signal Intensity}

The signal intensity measurements were highly reproducible (ICC $=0.909$ ). The timedependent changes in ACL or graft signal intensity are shown in Figure 3B. The ACL/graft signal intensities did not significantly change within the first two years in either group (BEAR: $\mathrm{F}=.7, \mathrm{df}=25.9, \mathrm{P}=.605$; ACLR: $\mathrm{F}=2.4, \mathrm{df}=32.2, \mathrm{P}=.075$ ). Both the BEAR and ACLR groups had a slightly higher mean ligament signal intensity at 3 months compared to the contralateral intact ACL, but these differences were not statistically significant ( $p>.4$; Figure 3B). The mean signal intensity of both the repaired ACLs and reconstructed grafts trended to a maximum at 6 months before reduction to the levels seen in their contralateral intact ACL at 24 months; though none of these changes were statistically significant (p>.1).

\section{Tunnel and Ligament Insertion Position}

For the repaired group, the $4.5 \mathrm{~mm}$ tunnels used to place the suture cinch were not located within the femoral or tibial footprints, consistent with the surgical protocol for tunnel placement. The femoral tunnels were located anterior and inferior (closer to the bottom of the condyle) to the native ACL insertion ( $\mathrm{p}<.02$; Figure S1-A and B). The $4.5 \mathrm{~mm}$ tibial tunnel for the suture cinch was also anterior to the tibial footprint of the native ACL ( $\mathrm{p}<.001$; Figure S1-C). The repaired ACL had the same sagittal elevation angle as the contralateral intact ACL (49.8 $\pm 3.2^{\circ}$ vs. $\left.49.8 \pm 1.7^{\circ} ; \mathrm{p}=.796\right)$ and coursed from insertion site to insertion site rather than from the femoral to tibia tunnel of the suture cinch. For the reconstructed group, the center of the $9 \mathrm{~mm}$ femoral tunnel was anterior to the center of the femoral insertion site ( $\mathrm{p}<.001$; Figure $\mathrm{S} 1-\mathrm{A})$ and in the same superior-inferior location as the native ACL insertion site ( $p=.795$; Figure $\mathrm{S} 1-\mathrm{B}$ ). The $9 \mathrm{~mm}$ tibial tunnels were slightly posterior to the tibial footprint of the native ACL ( $\mathrm{p}=.003$; Figure S1-C). The reconstructed grafts coursed from tibial to femoral tunnels and were more vertical than the contralateral intact ACL $\left(55.8 \pm 2.6^{\circ}\right.$ vs. $\left.49.3 \pm 1.0^{\circ} ; \mathrm{p}=.005\right)$.

\section{Associations between Anatomical Features of the Knee and Repaired ACL CSA and SI}

The regression analyses for the associations between the anatomical features of the knee and the repaired ACL cross-sectional area and normalized signal intensity at 24 months after surgery are presented in Table 2. For healing ligaments (BEAR group), the normalized femoral stump length, lateral tibial slope and medial tibial depth were significantly correlated with the signal intensity of the repaired ligament (Figure 4; $<<.05$ for all associations), while the pre-operative and post-operative notch size, and performance of a notchplasty, were predictive of the cross-sectional area of the repaired ligament at 24 months post-operatively (Figure 5; $\mathrm{p}<.05$ for all associations)).

A longer femoral stump prior to surgery was associated with a lower SI (closer to normal ligament) of the BEAR ligament (Figure 4A), as was a smaller lateral tibial slope and larger medial tibial depth (Figure 4B and 4C). A longer tibial stump was not associated with a lower SI or larger CSA (Table 2; p>.12).

The pre- and post-operative width of the notch as well as the extent of notchplasty (notch enlargement) correlated with increased cross-sectional area of the repaired ACL at 24 months (Figure 5). 


\section{Associations between Anatomical Features of the Knee and ACL Graft CSA and SI}

The regression analyses for the associations between the anatomical features of the knee and the ACL graft cross-sectional area and signal intensity at 24 months after surgery are presented in Table 3. None of the anatomical features of the knee were predictive of either graft cross-sectional area or signal intensity at 24 months post-operative.

\section{DISCUSSION}

Despite the similarities in patient reported outcomes, AP laxity of the knee and functional hop testing between the BEAR and ACLR procedures, ${ }^{38}$ this study identified differences in how the average cross-sectional area of the reconstructed graft and repaired ACL change within 2 years after ACL surgery. The observed changes in cross-sectional area and normalized signal intensity suggest that reconstructed grafts continue to remodel over the first 2 years after surgery, whereas the remodeling of the repaired ACL primarily happens within the first year. The results highlight the ability of the BEAR procedure to restore the signal intensity and anatomical (i.e. area and orientation) properties of the torn ACL to those of the native ACL, while ACLR resulted in significantly larger and more vertical grafts at 2 years. Unlike ACLR grafts, the repaired ACL size and normalized signal intensity at 24 months correlated with the pre-operative ACL femoral stump length, lateral tibial slope, medial tibial depth and femoral notch size.

The ACL grafts were significantly larger than the contralateral normal ACLs. This has been previously noted on MRIs performed three years post-operatively (>30\% larger). ${ }^{25,} 57,59$ Considering the cross-sectional area as a determinant of the maximum load a structure can bear, ${ }^{6,7}$ this increased cross-sectional area would predict the grafts would be able to support greater loads than the native ACL assuming equal material properties, and thus potentially decrease the risk of graft failure when a potentially injurious load is placed upon it. We were unable to determine if an increased cross-sectional area was associated with a lower failure rate as none of the subjects in this study had a ligament failure during the study; however, prior studies have reported that larger grafts promote a lower failure rate. ${ }^{13,32}$ Lastly, decreases in graft cross-sectional area within the first year after surgery have been previously reported $^{1}$; however, the reasons for the decrease in size of the graft over time is unknown, and whether this trend will continue requires a longer-term imaging study.

Increased normalized signal intensity has been associated with histologic changes in the ACL including the presence of inflammatory cells, actively synthesizing fibroblasts, and less collagen and blood vessel organization and orientation. ${ }^{9}$ Signal intensity measures, which reflect the "maturation" of ligament healing, have been used to evaluate outcomes and the factors that may influence outcomes following ACL reconstruction. ${ }^{26,29,30,42,44,51,52,56}$ In a cohort of ACL reconstructed patients, $\mathrm{Li}$ et al showed significant correlations between signal intensity and physical activity, and between signal intensity and time from surgery, both of which were sex dependent. ${ }^{29}$ Rose et al compared two types of allografts for ACL reconstruction using signal intensity. They determined that "graft maturity" was dependent on the position of the tibial tunnel and the sagittal plane orientation of the graft, though there were no differences between the two graft types (hamstring tendon vs tibialis anterior). ${ }^{51}$ Signal intensity measures have also been used to compare graft types, ${ }^{30,56}$ tunnel 
placement, ${ }^{26,52}$ and biologic augmentation. ${ }^{42,}{ }^{44}$ In this study, the signal intensity was similar in both groups, with a trend toward a higher signal intensity (more disorganized tissue) seen at 3 and 6 months, and normalization by 12 and 24 months. These observations are consistent with a prior preclinical study in repaired ligaments, which demonstrated an improvement in the histologic ligament maturity index after surgery. ${ }^{49}$ It is difficult to directly compare signal intensity values across studies as the signal intensity magnitude is dependent of the hardware, sequence, sequence parameters and the normalization process selected, ${ }^{8}$ which vary across studies. This is a limitation of using signal intensity as an outcome measure, particularly for multicenter studies. Work is currently under way to implement and optimize relaxometry methods (e.g., $\mathrm{T}_{2}{ }^{*}$ relaxation time), ${ }^{2,3,7,9}$ which are theoretically less dependent on hardware and post-processing and would provide consistency across platforms, institutions and trials. ${ }^{8}$ While the current study used a different imaging protocol, normalization structure and analysis technique from prior clinical studies of ACL reconstruction, the overall finding of higher signal intensity early on followed by normalization is consistent with prior reports of temporal changes in ACL graft signal intensity within 1 year after ACL reconstruction, ${ }^{28}$ decreasing signal intensity of grafts as the post-operative interval increases, ${ }^{29,43}$ and decreased metabolic activity of the grafts as assessed by PET-MRI. ${ }^{50}$ Moreover, normalized signal intensity measures obtained using the same hardware and sequence within a study provides insight into the integrity of the healing ligament or graft.

The more vertical sagittal orientation seen in the ACL grafts in this study is consistent with that previously observed for ACL grafts placed using an anteromedial portal technique (55 degrees for grafts placed using an anteromedial portal and 64 degrees when a transtibial technique is used ${ }^{58}$ and has also been associated with increased cartilage damage in preclinical models. ${ }^{22}$ Interestingly, the non-anatomic tunnel placement for the suture cinch used in the BEAR subjects did not alter the sagittal orientation of the healing ACL, which healed with a sagittal orientation identical to that of the native ACL. On MRI, the repaired ACL was found to course from insertion site to insertion site, rather than from the femoral cinch tunnel to the tibial cinch tunnel. The insertion to insertion healing also suggests that the BEAR procedure may preserve the anatomy of the ACL footprints, which has been shown to influence ACL tension patterns and knee biomechanics. ${ }^{24}$

While none of the anatomical parameters measured here influenced either the graft crosssectional area or signal intensity, there were several parameters that significantly affected the healing ACL, including modifiable factors (notch size), non-modifiable factors (femoral stump length, medial tibial depth) and potentially modifiable factors (lateral tibial slope). A greater notch width was associated with bigger repaired ACL cross-sectional area. A large notch can be indicative of a larger native ACL, which can ultimately lead to a larger repaired ACL. Performance of a notchplasty had a reasonably large effect on the cross-sectional area of the healing ACL, with every mm of notchplasty leading to a $3 \mathrm{~mm}^{2}(6 \%)$ increase in ACL cross-sectional area. This finding was most evident by observed variations in repaired ACL cross-sectional area which was strongly correlated with the side range of post-operative notch width and notchplasty as shown in Figure 5. As cross-sectional area is a primary determinant of the maximum load that can be withstood by the ACL, performance of a notchplasty when performing a BEAR procedure may be a reasonable surgical choice; 
however, further studies to determine the long-term outcomes of adding this to the procedure are needed.

Smaller femoral stump length, shallower medial tibial depth and steeper posterior slope of the lateral tibial plateau resulted in significant increases in normalized signal intensity of the healing ACL. Although these features are not easily modifiable, they may potentially be considered as biomarkers to predict the risk of re-injury ${ }^{27,53}$ and inferior post-operative outcomes. ${ }^{23}$ While the tibial stump is pulled towards the femur into the scaffold using sutures, the femoral stump is not tensioned during the BEAR procedure. Thus, a longer femoral stump may more easily provide a greater length of low intensity ACL fibers in the gap between the insertion points, leading to a more organized (lower signal intensity) healing ACL. Lateral tibial slope and medial tibial depth have both been shown to influence knee biomechanics ${ }^{19,34,35}$ and ACL loading. ${ }^{31,35}$ A steep lateral tibial slope and shallow medial tibial depth have previously been associated with increased risk of ACL injury. ${ }^{4}, 20$ Increased lateral tibial slope and decreased medial depth can lead to additional anteroposterior $^{21,40}$ and rotational ${ }^{21,26,59}$ instability, and increased ACL loading, which may be detrimental to collagen organization in the early phases of healing after bridgeenhanced ACL repair. If this last hypothesis were verified, the risks associated with a higher tibial slope might be mitigated by utilizing a more conservative rehabilitation protocol for these subjects. Further work to investigate this hypothesis is needed.

There were several limitations to this study. The first was the small sample size in each group. This study was a first-in-human safety study of a new medical device. FDA approval for these initial safety studies is typically limited to studies with a small sample size, such that few subjects will suffer an adverse event if the device is found to have a high rate of failure or infection. Second, the total ACL length was quantified from the preoperative MRIs. While we attempted to scan all knees in a standardize way, small differences in knee subluxation may have resulted in measurement errors in quantifying total ACL length. Third, while the association of healing ACL cross sectional area and preoperative notch width appears to rely on the two patients with very large notch widths (Fig 5A), the associations to postoperative notch width (Fig 5B) and notchplasty (Fig 5C) better depict the nature of these relationships by demonstrating small ACL area in patients with smaller notch widths and less notchplasty, and largest ACL area in those with bigger notch widths and more notchplasty. These graphs also show how patients with average notch and notchplasty had average ACL cross-sectional area. Fourth, the contralateral ACL intact knee was only imaged at 2 years due to imaging time constraints. Longitudinal assessment of the contralateral knee could have helped to better investigate the time and treatment dependent changes in treated ACL. Lastly, this was a cohort study, rather than a randomized control trial. This was also due to the fact that this was a first-in-human trial, and the study team and surgeons felt there was not sufficient equipoise to ethically conduct a randomized control trial. Thus, the subjects in the trial were allowed to choose the treatment arm they wished to be in, a study design that has the potential to introduce a selection bias. Further studies of a larger number of subjects with a randomized control design are planned.

In conclusion, post-operative MR imaging of healing ACL grafts and repaired ACLs suggests that ACL grafts with autograft hamstring tendon are larger and more vertically 
oriented than both the native ACL and the repaired ACL. The time-dependent changes in signal intensity suggest the repaired ligaments and grafts both undergo a period of maturation, with both groups attaining average signal intensities that were similar to the contralateral intact ACL by 12 (repaired group) and 24 (ACL reconstruction group) months after surgery. The results here also suggest that the cross-sectional area of the repaired ACL may be affected by notch width, while the signal intensity of the repaired ACLs appeared to be correlated with specific anatomic features, observations that deserve further study in a larger cohort of subjects. These findings suggest that MRI techniques can be used to noninvasively monitor the healing and maturation of both ACL grafts and repaired ACLs after surgery.

\section{Supplementary Material}

Refer to Web version on PubMed Central for supplementary material.

\section{ACKNOWLEDEGEMENTS:}

We would like to acknowledge the significant contributions of the clinical trial team including Bethany Trainor, Andrea Hale and Shanika Coney. We would also like to acknowledge the contributions of our medical safety monitoring team of Joseph DeAngelis and Peter Nigrovic, our data monitors Margaret Malsch and Megan Fitzgerald, as well as the clinical care team for the trial patients, including Kathryn Ackerman, Alyssa Aguiar, Judd Allen, Michael Beasley, Jennifer Beck, Alison Biercevicz, Dennis Borg, Nicole Bottino, Stephanie Burgess, Melissa Christino, Andrea Cianci, Sarah Collins, Gianmichel Corrado, Sara Cline, Corey Dawkins, Pierre D'Hemecourt, Peter Fabricant, Jon Ferguson, Michele Flannery, Joseph Founds, Casey Gavin, Ellen Geminiani, Stacey Gigante, Annie Griffin, Emily Hanson, Elspeth Hart, Pamela Horne-Goffigan, Leslie Kalish, Elizabeth KillKelly, Elizabeth Kramer, Pamela Lang, Chaimae Martin, Michael McClincy, William Meehan, Ariana Moccia, Mariah Mullen, Stacey Murphy, Emily Niu, Michael O’Brien, Nikolas Paschos, Katrina Plavetsky, Bridget Quinn, Lauren Redler, Shannon Savage, Edward Schleyer, Benjamin Shore, Cynthia Stein, Andrea Stracciolini, Dai Sugimoto, Dylan Taylor, Ashleigh Thorogood, Natasha Trentacosta, Patrick Vavken, Lisa Vopat and Lenise Young. We would also like to acknowledge the efforts of the scaffold manufacturing team, including Benedikt Proffen, Gabe Perrone, Gordon Roberts, Doris Peterkin and Jakob Sieker. We are also grateful for the study design guidance provided by the Division of Orthopedic Devices at the Center for Devices and Radiological Health at the U.S. Food and Drug Administration, particularly the efforts of Jemin Dedania and Neil Barkin. Lastly, we would like to acknowledge funding support from the Translational Research Program at Boston Children's Hospital, the Boston Children's Hospital Orthopaedic Surgery Foundation, the Boston Children's Hospital Sports Medicine Foundation and the National Football League Players Association through the Harvard Catalyst's Football Players Health Study, as well as the National Institutes of Health and the National Institute of Arthritis and Musculoskeletal and Skin Diseases through grant numbers R01-AR065462 and R01-AR056834.

\section{REFERENCES}

1. Astur DC, Arliani GG, Debieux P, Kaleka CC, Amaro JT, Cohen M. Intraarticular hamstring graft diameter decreases with continuing knee growth after ACL reconstruction with open physes. Knee Surg Sports Traumatol Arthrosc. 2016;24(3):792-795. [PubMed: 26860290]

2. Beveridge JE, Machan JT, Walsh EG, et al. Magnetic resonance measurements of tissue quantity and quality using $\mathrm{T} 2 *$ relaxometry predict temporal changes in the biomechanical properties of the healing ACL. J Orthop Res. 2018;36(6):1701-1709. [PubMed: 29227559]

3. Beveridge JE, Walsh EG, Murray MM, Fleming BC. Sensitivity of ACL volume and T2* relaxation time to magnetic resonance imaging scan conditions. J Biomech. 2017;56:117-121. [PubMed: 28359570]

4. Beynnon BD, Hall JS, Sturnick DR, et al. Increased slope of the lateral tibial plateau subchondral bone is associated with greater risk of noncontact ACL injury in females but not in males: a prospective cohort study with a nested, matched case-control analysis. Am J Sports Med. 2014;42(5):1039-1048. [PubMed: 24590006] 
5. Biercevicz AM, Akelman MR, Fadale PD, et al. MRI volume and signal intensity of ACL graft predict clinical, functional, and patient-oriented outcome measures after ACL reconstruction. Am J Sports Med. 2015;43(3):693-699. [PubMed: 25540298]

6. Biercevicz AM, Miranda DL, Machan JT, Murray MM, Fleming BC. In situ, noninvasive, T2*weighted MRI-derived parameters predict ex vivo structural properties of an anterior cruciate ligament reconstruction or bioenhanced primary repair in a porcine model. Am J Sports Med. 2013;41(3):560-566. [PubMed: 23348076]

7. Biercevicz AM, Murray MM, Walsh EG, Miranda DL, Machan JT, Fleming BC. T2 * MR relaxometry and ligament volume are associated with the structural properties of the healing ACL. J Orthop Res. 2014;32(4):492-499. [PubMed: 24338640]

8. Biercevicz AM, Murray MM, Walsh EG, Miranda DL, Machan JT, Fleming BC. T2* MR relaxometry and ligament volume are associated with the structural properties of the healing ACL. J Orthop Res. 2014;32(4):492-499. [PubMed: 24338640]

9. Biercevicz AM, Proffen BL, Murray MM, Walsh EG, Fleming BC. T2* relaxometry and volume predict semi-quantitative histological scoring of an ACL bridge-enhanced primary repair in a porcine model. J Orthop Res. 2015;33(8):1180-1187. [PubMed: 25764143]

10. Christino MA, Fantry AJ, Vopat BG. Psychological Aspects of Recovery Following Anterior Cruciate Ligament Reconstruction. J Am Acad Orthop Surg. 2015;23(8):501-509. [PubMed: 26209145]

11. Christino MA, Fleming BC, Machan JT, Shalvoy RM. Psychological Factors Associated With Anterior Cruciate Ligament Reconstruction Recovery. Orthop J Sports Med. 2016;4(3): 2325967116638341.

12. Cohen SB, VanBeek C, Starman JS, Armfield D, Irrgang JJ, Fu FH. MRI measurement of the 2 bundles of the normal anterior cruciate ligament. Orthopedics. 2009;32(9).

13. Conte EJ, Hyatt AE, Gatt CJ Jr., Dhawan A Hamstring autograft size can be predicted and is a potential risk factor for anterior cruciate ligament reconstruction failure. Arthroscopy. 2014;30(7): 882-890. [PubMed: 24951356]

14. Eliasziw M, Young SL, Woodbury MG, Fryday-Field K. Statistical methodology for the concurrent assessment of interrater and intrarater reliability: using goniometric measurements as an example. Phys Ther. 1994;74(8):777-788. [PubMed: 8047565]

15. Feagin JR JA, Curl WW. Isolated tear of the anterior cruciate ligament: 5-year follow-up study. The American journal of sports medicine. 1976;4(3):95-100. [PubMed: 984289]

16. Forsythe B, Kopf S, Wong AK, et al. The location of femoral and tibial tunnels in anatomic double-bundle anterior cruciate ligament reconstruction analyzed by three-dimensional computed tomography models. J Bone Joint Surg Am. 2010;92(6):1418-1426. [PubMed: 20516317]

17. Geeslin AG, Chahla J, Moatshe G, et al. Anterolateral Knee Extra-articular Stabilizers: A Robotic Sectioning Study of the Anterolateral Ligament and Distal Iliotibial Band Kaplan Fibers. Am J Sports Med. 2018;46(6):1352-1361. [PubMed: 29558208]

18. Group MK, Spindler KP, Huston LJ, et al. Ten-Year Outcomes and Risk Factors After Anterior Cruciate Ligament Reconstruction: A MOON Longitudinal Prospective Cohort Study. Am J Sports Med. 2018;46(4):815-825. [PubMed: 29543512]

19. Hashemi J, Chandrashekar N, Gill B, et al. The geometry of the tibial plateau and its influence on the biomechanics of the tibiofemoral joint. J Bone Joint Surg Am. 2008;90(12):2724-2734. [PubMed: 19047719]

20. Hashemi J, Chandrashekar N, Mansouri H, et al. Shallow medial tibial plateau and steep medial and lateral tibial slopes: New risk factors for anterior cruciate ligament injuries. Am J Sports Med. 2010;38(1):54-62. [PubMed: 19846692]

21. Hudek R, Schmutz S, Regenfelder F, Fuchs B, Koch PP. Novel measurement technique of the tibial slope on conventional MRI. Clin Orthop Relat Res. 2009;467(8):2066-2072. [PubMed: 19190973]

22. Kiapour AM, Fleming BC, Murray MM. Structural and Anatomic Restoration of the Anterior Cruciate Ligament Is Associated With Less Cartilage Damage 1 Year After Surgery: Healing Ligament Properties Affect Cartilage Damage. Orthop J Sports Med. 2017;5(8): 2325967117723886. 
23. Kiapour AM, Yang DS, Badger GJ, et al. Anatomic Features of the Tibial Plateau Predict Outcomes of ACL Reconstruction Within 7 Years After Surgery. The American journal of sports medicine. 2019:0363546518823556.

24. Kopf S, Musahl V, Tashman S, Szczodry M, Shen W, Fu FH. A systematic review of the femoral origin and tibial insertion morphology of the ACL. Knee Surg Sports Traumatol Arthrosc. 2009;17(3):213-219. [PubMed: 19139847]

25. Lee BH, Seo DY, Bansal S, Kim JH, Ahn JH, Wang JH. Comparative Magnetic Resonance Imaging Study of Cross-Sectional Area of Anatomic Double Bundle Anterior Cruciate Ligament Reconstruction Grafts and the Contralateral Uninjured Knee. Arthroscopy. 2016;32(2):321-329 e321. [PubMed: 26603824]

26. Lee DW, Kim JG, Lee JH, Park JH, Kim DH. Comparison of modified transtibial and outside-in techniques in anatomic single-bundle anterior cruciate ligament reconstruction. Arthroscopy. 2018;34(10):2857-2870. [PubMed: 30197202]

27. Levins JG, Sturnick DR, Argentieri EC, et al. Geometric Risk Factors Associated With Noncontact Anterior Cruciate Ligament Graft Rupture. Am J Sports Med. 2016;44(10):2537-2545. [PubMed: 27514738]

28. Li H, Chen J, Li H, Wu Z, Chen S. MRI-based ACL graft maturity does not predict clinical and functional outcomes during the first year after ACL reconstruction. Knee Surg Sports Traumatol Arthrosc. 2017;25(10):3171-3178. [PubMed: 27485123]

29. Li H, Chen S, Tao H, Li H, Chen S. Correlation analysis of potential factors influencing graft maturity after anterior cruciate ligament reconstruction. Orthop J Sports Med. 2014;2(10): 2325967114553552.

30. Li H, Tao H, Cho S, Chen S, Yao Z, Chen S. Difference in graft maturity of the reconstructed anterior cruciate ligament 2 years postoperatively: a comparison between autografts and allografts in young men using clinical and 3.0-T magnetic resonance imaging evaluation. Am J Sports Med. 2012;40(7):1519-1526. [PubMed: 22495290]

31. Lipps DB, Oh YK, Ashton-Miller JA, Wojtys EM. Morphologic characteristics help explain the gender difference in peak anterior cruciate ligament strain during a simulated pivot landing. Am J Sports Med. 2012;40(1):32-40. [PubMed: 21917612]

32. Magnussen RA, Lawrence JT, West RL, Toth AP, Taylor DC, Garrett WE. Graft size and patient age are predictors of early revision after anterior cruciate ligament reconstruction with hamstring autograft. Arthroscopy. 2012;28(4):526-531. [PubMed: 22305299]

33. Magnussen RA, Reinke EK, Huston LJ, Group M, Hewett TE, Spindler KP. Factors Associated With High-Grade Lachman, Pivot Shift, and Anterior Drawer at the Time of Anterior Cruciate Ligament Reconstruction. Arthroscopy. 2016;32(6):1080-1085. [PubMed: 26821957]

34. McLean SG, Lucey SM, Rohrer S, Brandon C. Knee joint anatomy predicts high-risk in vivo dynamic landing knee biomechanics. Clin Biomech (Bristol, Avon). 2010;25(8):781-788.

35. McLean SG, Oh YK, Palmer ML, et al. The relationship between anterior tibial acceleration, tibial slope, and ACL strain during a simulated jump landing task. J Bone Joint Surg Am. 2011;93(14): 1310-1317. [PubMed: 21792497]

36. Murray MM, Fleming BC. Biology of anterior cruciate ligament injury and repair: Kappa delta ann doner vaughn award paper 2013. J Orthop Res. 2013;31(10):1501-1506. [PubMed: 23818453]

37. Murray MM, Fleming BC. Use of a bioactive scaffold to stimulate anterior cruciate ligament healing also minimizes posttraumatic osteoarthritis after surgery. Am J Sports Med. 2013;41(8): 1762-1770. [PubMed: 23857883]

38. Murray MM, Flutie BM, Kalish LA, et al. The Bridge-Enhanced Anterior Cruciate Ligament Repair (BEAR) Procedure: An Early Feasibility Cohort Study. Orthop J Sports Med. 2016;4(11): 2325967116672176.

39. Murray MM, Kalish LA, Fleming BC, et al. Bridge-Enhanced Anterior Cruciate Ligament Repair: Two-Year Results of a First-in-Human Study. Orthopaedic Journal of Sports Medicine. 2019;7(3): 2325967118824356.

40. Murray MM, Magarian EM, Harrison SL, Mastrangelo AN, Zurakowski D, Fleming BC. The effect of skeletal maturity on functional healing of the anterior cruciate ligament. The Journal of Bone and Joint Surgery. American volume. 2010;92(11):2039. 
41. Musahl V, Ayeni OR, Citak M, Irrgang JJ, Pearle AD, Wickiewicz TL. The influence of bony morphology on the magnitude of the pivot shift. Knee Surg Sports Traumatol Arthrosc. 2010;18(9):1232-1238. [PubMed: 20376621]

42. Naraoka T, Kimura Y, Tsuda E, Yamamoto Y, Ishibashi Y. Is remnant preservation truly beneficial to anterior cruciate ligament reconstruction healing? Am J Sports Med. 2017:363546516682241.

43. Ntoulia A, Papadopoulou F, Zampeli F, Ristanis S, Argyropoulou M, Georgoulis A. Evaluation with contrast-enhanced magnetic resonance imaging of the anterior cruciate ligament graft during its healing process: a two-year prospective study. Skeletal Radiol. 2013;42(4):541-552. [PubMed: 23229627]

44. Orrego M, Larrain C, Rosales J, et al. Effects of platelet concentrate and a bone plug on the healing of hamstring tendons in a bone tunnel. Arthroscopy. 2008;24(12):1373-1380. [PubMed: 19038708]

45. Park JS, Nam DC, Kim DH, Kim HK, Hwang SC. Measurement of Knee Morphometrics Using MRI: A Comparative Study between ACL-Injured and Non-Injured Knees. Knee Surg Relat Res. 2012;24(3):180-185. [PubMed: 22977796]

46. Paterno MV, Flynn K, Thomas S, Schmitt LC. Self-Reported Fear Predicts Functional Performance and Second ACL Injury After ACL Reconstruction and Return to Sport: A Pilot Study. Sports Health. 2018;10(3):228-233. [PubMed: 29272209]

47. Perrone GS, Proffen BL, Kiapour AM, Sieker JT, Fleming BC, Murray MM. Bench-to-bedside: Bridge-enhanced anterior cruciate ligament repair. J Orthop Res. 2017;35(12):2606-2612. [PubMed: 28608618]

48. Proffen B, Perrone G, Roberts G, Murray M. Bridge-enhanced ACL repair: A review of the science and the pathway through FDA investigational device approval. Ann Biomed Eng. 2015;43(3):805818. [PubMed: 25631206]

49. Proffen BL, Fleming BC, Murray MM. Histological Predictors of Maximum Failure Loads Differ Between the Healing ACL and ACL Grafts After 6 and 12 Months In Vivo. Orthopaedic Journal of Sports Medicine. 2013;1(6):2325967113512457.

50. R AM, Binzel K, Zhang J, et al. ACL graft metabolic activity assessed by (18)FDG PET-MRI. Knee. 2017;24(4):792-797. [PubMed: 28559006]

51. Rose M, Crawford D. Allograft maturation after reconstruction of the anterior cruciate ligament is dependent on graft parameters in the sagittal plane. Orthop J Sports Med. 2017;5(8): 2325967117719695.

52. Saito M, Nakajima A, Sonobe M, et al. Superior graft maturation after anatomical double-bundle anterior cruciate ligament reconstruction using the transtibial drilling technique compared to the transportal technique. Knee Surg Sports Traumatol Arthrosc. 2018.

53. Salmon LJ, Heath E, Akrawi H, Roe JP, Linklater J, Pinczewski LA. 20-Year Outcomes of Anterior Cruciate Ligament Reconstruction With Hamstring Tendon Autograft: The Catastrophic Effect of Age and Posterior Tibial Slope. Am J Sports Med. 2018;46(3):531-543. [PubMed: 29244525]

54. Scanlan SF, Blazek K, Chaudhari AM, Safran MR, Andriacchi TP. Graft orientation influences the knee flexion moment during walking in patients with anterior cruciate ligament reconstruction. Am J Sports Med. 2009;37(11):2173-2178. [PubMed: 19729363]

55. Shybut TB, Vega CE, Haddad J, et al. Effect of lateral meniscal root tear on the stability of the anterior cruciate ligament-deficient knee. Am J Sports Med. 2015;43(4):905-911. [PubMed: 25589386]

56. Tanaka Y, Yonetani Y, Shiozaki Y, et al. MRI analysis of single-, double-, and triple-bundle anterior cruciate ligament grafts. Knee Surg Sports Traumatol Arthrosc. 2014;22(7):1541-1548. [PubMed: 23749216]

57. Thein R, Spitzer E, Doyle J, et al. The ACL Graft Has Different Cross-sectional Dimensions Compared With the Native ACL: Implications for Graft Impingement. Am J Sports Med. 2016;44(8):2097-2105. [PubMed: 27179055]

58. Vermesan D, Inchingolo F, Patrascu J, et al. Anterior cruciate ligament reconstruction and determination of tunnel size and graft obliquity. Eur Rev Med Pharmacol Sci. 2015;19(3):357364. [PubMed: 25720703] 
59. Vermesan D, Prejbeanu R, Trocan I, et al. Reconstructed ACLs have different cross-sectional areas compared to the native contralaterals on postoperative MRIs. A pilot study. Eur Rev Med Pharmacol Sci. 2015;19(7):1155-1160. [PubMed: 25912573]

60. Whitney DC, Sturnick DR, Vacek PM, et al. Relationship between the risk of suffering a first-time noncontact ACL injury and geometry of the femoral notch and ACL: A prospective cohort study with a nested case-control analysis. Am J Sports Med. 2014;42(8):1796-1805. [PubMed: 24866891] 


\section{What is known about the subject:}

Magnetic resonance (MR) imaging has been used in several preclinical and clinical studies to evaluate ACL/graft healing and associated outcomes after surgery. Due to its noninvasive and nondestructive nature, MR imaging can be used to study the timedependent changes in ligament structure after surgery. This approach could be used to compare different surgical procedures, or to evaluate factors influencing ligament healing. Bridge-Enhanced ACL Repair (BEAR ${ }^{\circledR}$ ) is a new technique being developed for clinical use. It has shown to have a reasonable safety profile and early efficacy studies are encouraging. A recent study has shown comparable short-term outcomes (i.e. patientreported, clinical and functional) between BEAR and ACL reconstruction procedures. However, the structural changes in healing ACL compared to the reconstructed graft and the factors which may influence the size and quality of the healing ligament are not clearly understood.

\section{What this manuscript adds to the literature:}

The current study shows that cross-sectional area and signal intensity (a measure of tissue quality) of both the repaired ACL and reconstructed graft undergo significant changes within the first 2 years after ACL surgery. BEAR can successfully restore the size, signal intensity and sagittal orientation of the torn ACL to levels seen in the contralateral intact ACL. These properties of the repaired ACL are influenced by the location of the ACL tear as well as key morphologic features of the femur (i.e. notch width) and tibia (lateral tibial slope and medial tibial depth). MRI can be used to better understand ACL healing and remodeling after surgery and to identify the factors influencing this process. 


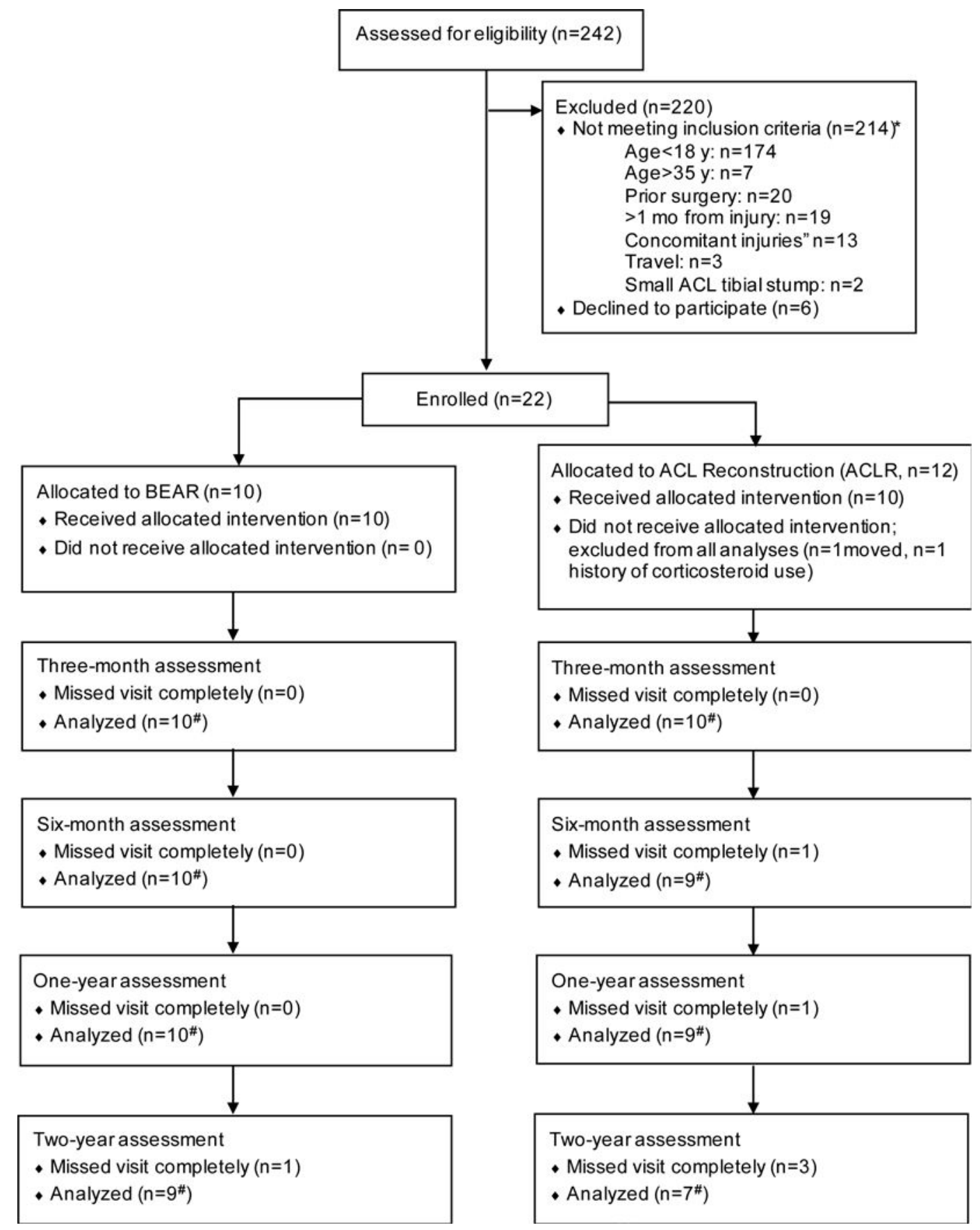

Figure 1.

Flow diagram of patient recruitment and follow up. ${ }^{38} *$ The total number of patients not meeting inclusion criteria totals to greater than 214 , as some patients met more than 1 exclusion criterion. "Not all patients completed every component of the follow-up assessments. Sample sizes with data available for analysis for each component are shown in the figures and tables. 

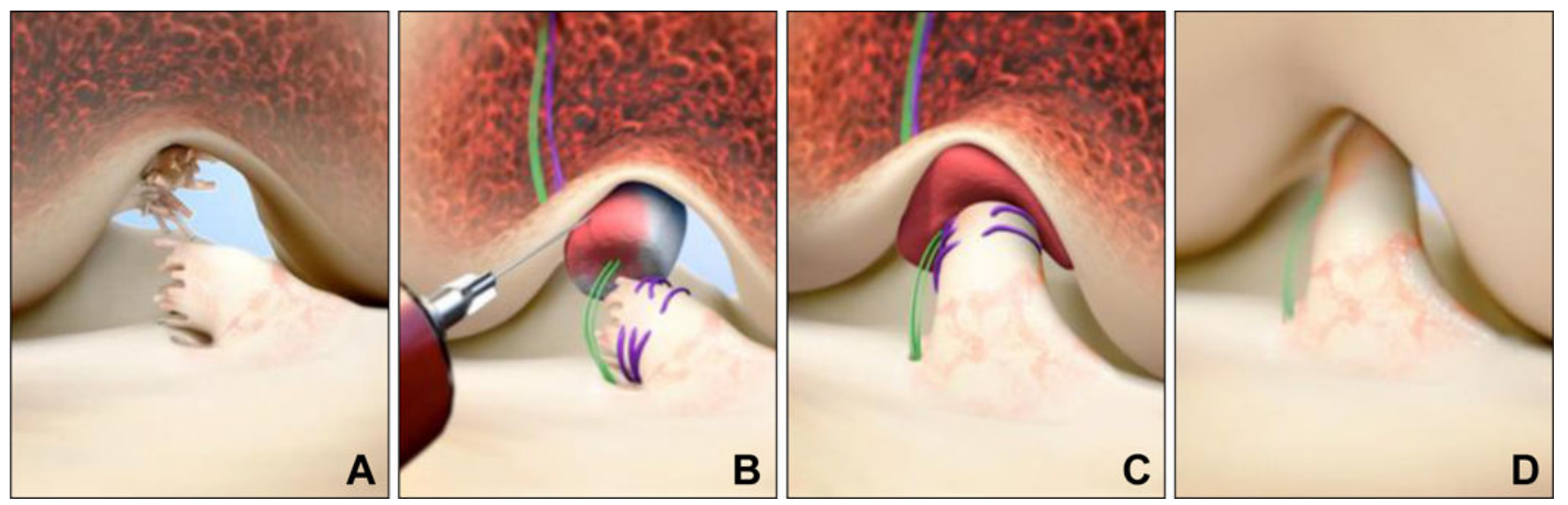

Figure 2.

Stepwise demonstration of the "Bridge-Enhanced ACL Repair" technique using the scaffold. In this technique, the torn ACL tissue is preserved (A). A whip stitch of \#2 absorbable suture (purple suture) is placed into the tibial stump of the ACL. Small tunnels $(4 \mathrm{~mm})$ are drilled in the femur and tibia and a suture cinch comprised of a cortical button with two \#2 nonabsorbable sutures (green sutures) and the \#2 absorbable ACL sutures are passed through the femoral tunnel and the button engaged on the proximal femoral cortex. The non-absorbable sutures are threaded through the scaffold, tibial tunnel and secured in place with a second extracortical button. The scaffold is then saturated with $10 \mathrm{~mL}$ of the patient's blood (B), and the tibial stump pulled up into the saturated scaffold (C). The ends of the torn ACL then grow into the scaffold and the ligament reunites (D). Used with permission from Murray et al, OJSM $2016 .^{38}$ 


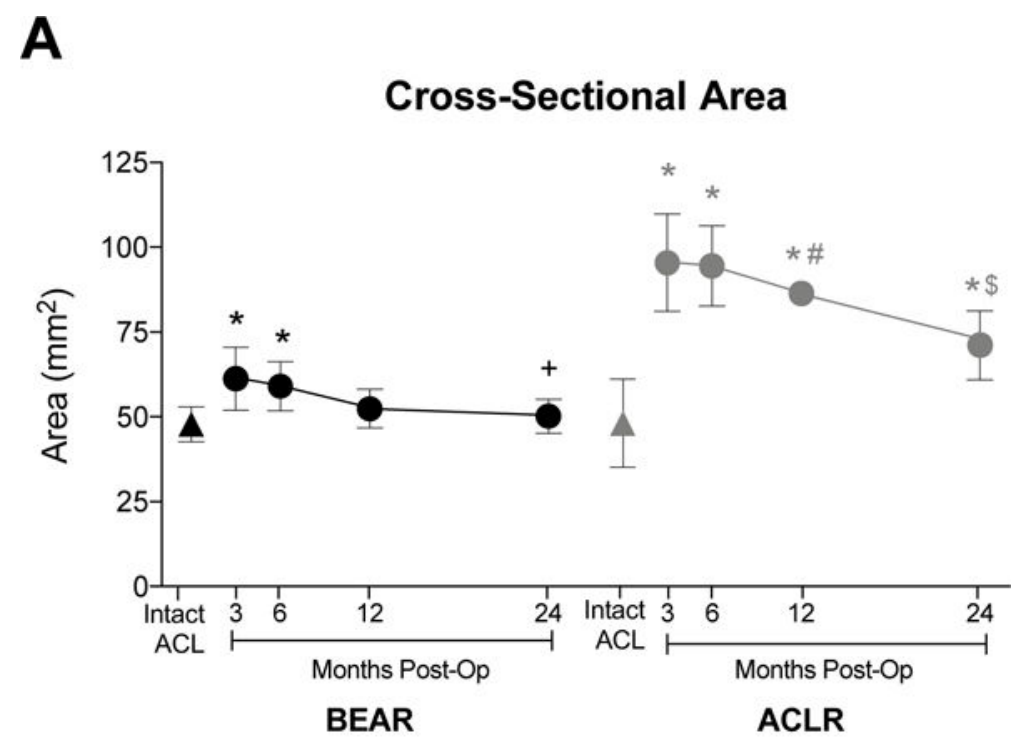

B

\section{Signal Intensity}

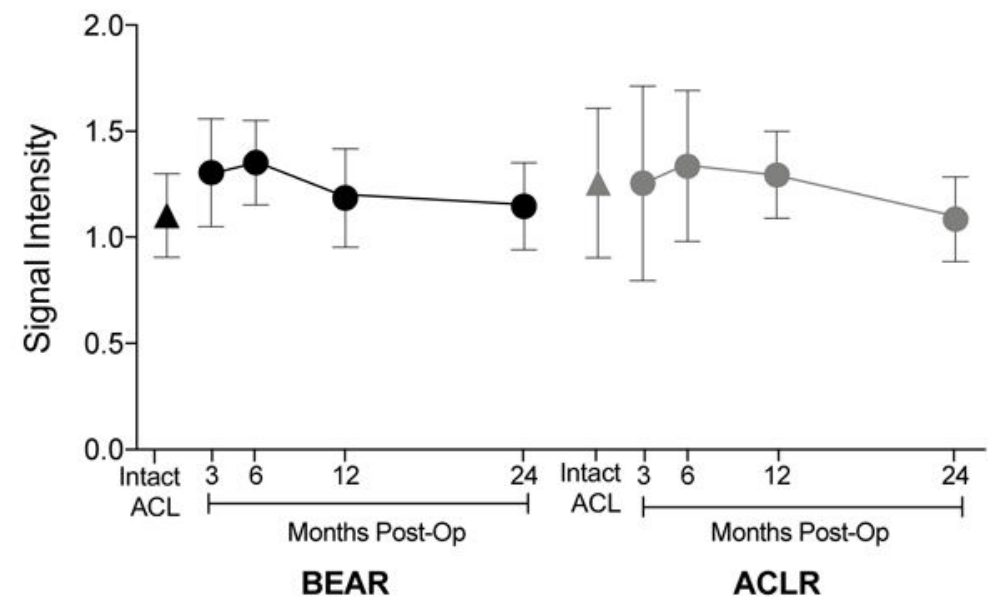

\begin{tabular}{|c|c|c|c|c|c|}
\hline & \multirow{2}{*}{$\begin{array}{l}\text { Intact } \\
\mathrm{ACL}\end{array}$} & \multicolumn{4}{|c|}{ Months Post-Op } \\
\hline & & 3 & 6 & 12 & 24 \\
\hline BEAR & $N=7$ & $\mathrm{~N}=10$ & $\mathrm{~N}=10$ & $\mathrm{~N}=9$ & $\mathrm{~N}=9$ \\
\hline ACLR & $\mathrm{N}=7$ & $\mathrm{~N}=10$ & $\mathrm{~N}=8$ & $\mathrm{~N}=6$ & $\mathrm{~N}=7$ \\
\hline
\end{tabular}

Figure 3.

Time-dependent changes in ACL/graft (A) cross-sectional area and (B) signal intensity. (C) Sample size for each treatment group at every time point. Intact ACL values are from the contralateral intact knee imaged at 24 months. Mean \pm standard deviation. ${ }^{*} \mathrm{p}<.02$ for comparisons to the contralateral intact ACL. +Smaller repaired ACL cross-sectional area at 24 months compared to 3 months $(\mathrm{p}=.023)$. \$ Smaller reconstructed graft cross-sectional area at 24 months compared to $3(\mathrm{p}<.001)$ and $6(\mathrm{p}=.002)$ months. ${ }^{\#}$ Standard deviations are 
smaller than the symbol $\left( \pm 2.2 \mathrm{~mm}^{2}\right)$. BEAR: Bridge-Enhanced ACL Repair; ACLR: ACL reconstruction. 

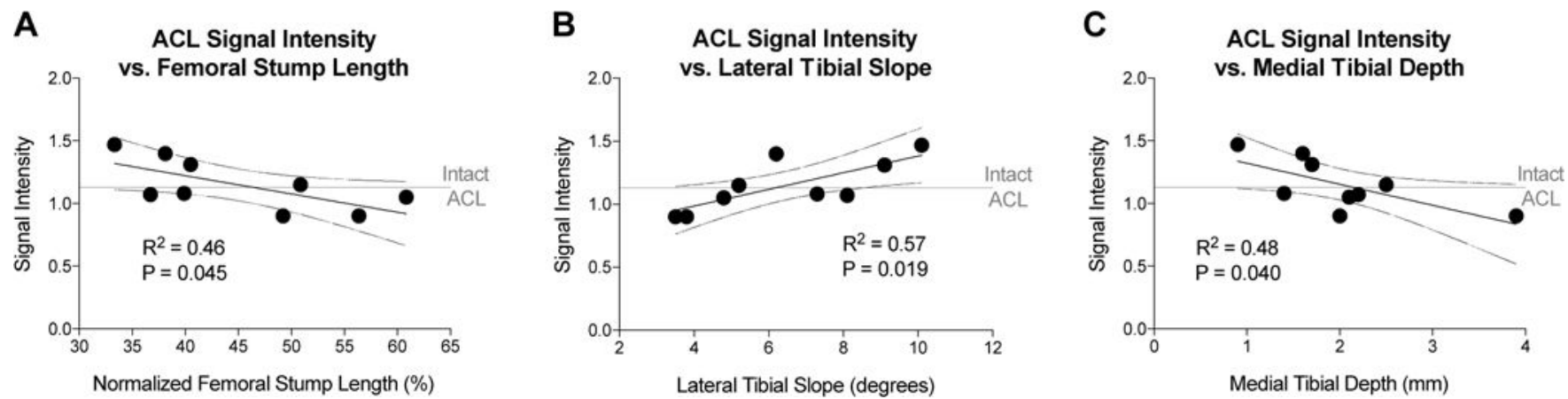

Figure 4:

Associations between BEAR ACL signal intensity at 24 months with (A) femoral stump length (normalized to ACL length), (B) lateral tibial slope, and (C) medial tibial depth. $\mathrm{N}=$ 9. Dotted lines represent the $95 \%$ confidence intervals. Horizontal line represents the average signal intensity of the contralateral intact ACL. 

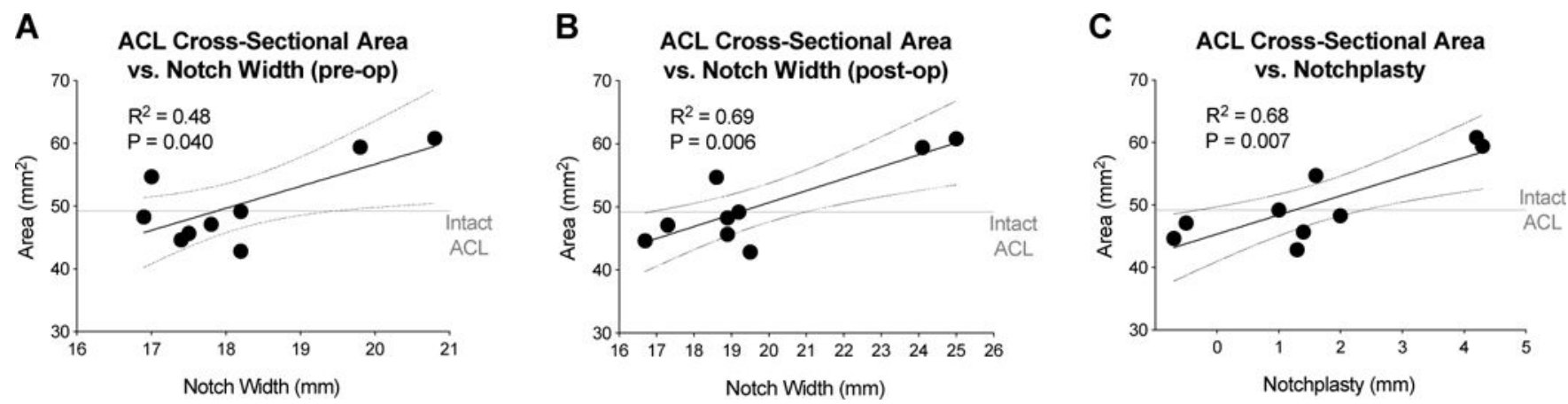

Figure 5:

Associations between ACL cross-sectional area of the BEAR group at 24 months and preoperatively notch width $(\mathrm{A})$ and post-operative notch width $(\mathrm{B})$, and $(\mathrm{C})$ notchplasty. $\mathrm{N}=9$. Dotted lines represent the $95 \%$ confidence intervals. Horizontal line represents the average cross-sectional area of the contralateral intact ACL. 
Table 1.

Anatomical features as determined from the MR scans for the subjects undergoing Bridge-enhanced ACL Repair (BEAR) and ACL Reconstruction with autograft hamstring graft (ACLR).

\begin{tabular}{lccccccc}
\hline \multirow{2}{*}{ Measurement } & \multicolumn{2}{c}{ BEAR } & & \multicolumn{2}{c}{ ACLR } & P \\
\cline { 2 - 3 } & Mean $( \pm$ SD) & $\mathbf{N}$ & & Mean $( \pm$ SD $)$ & N & Value \\
\hline${ }^{a}$ Normalized Femoral Stump Length, $\%$ & $45.6( \pm 10.9)$ & 10 & $38.8( \pm 7.3)$ & 10 & .117 \\
${ }^{a}$ Normalized Tibial Stump Length, $\%$ & $49.4( \pm 11.4)$ & 10 & $53.9( \pm 11.4)$ & 10 & .361 \\
Lateral Tibial Slope, degrees & $6.1( \pm 2.5)$ & 10 & $6.9( \pm 2.1)$ & 10 & .439 \\
Medial Tibial Slope, degrees & $4.6( \pm 2.5)$ & 10 & $5.9( \pm 2.7)$ & 10 & .291 \\
Medial Tibial Depth, mm & $2.0( \pm 0.8)$ & 10 & $2.0( \pm 0.7)$ & 10 & .881 \\
Notch Width (pre-operative), mm & $18.6( \pm 1.9)$ & 10 & $18.8( \pm 3.2)$ & 10 & .888 \\
\hline
\end{tabular}

a Normalized to the ACL length

$\mathrm{SD}$, standard deviation.

BEAR, Bridge-enhanced ACL repair

ACLR, ACL reconstruction. 
Table 2:

Regression coefficients for the MRI-based predictors of the repaired ACL cross-sectional area/signal intensity at 24 months. Significant associations are highlighted in bold. $\beta$ is regression slope, representing change in the outcome per unit change in the predictor.

\begin{tabular}{|c|c|c|c|c|c|c|}
\hline \multirow[b]{3}{*}{ MRI-based Predictors } & \multicolumn{6}{|c|}{ Repaired ACL Properties (24 months) } \\
\hline & \multicolumn{3}{|c|}{ Cross-Sectional Area } & \multicolumn{3}{|c|}{ Signal Intensity } \\
\hline & $\begin{array}{c}\beta \\
(95 \% \mathrm{CI})\end{array}$ & $\mathbf{R}^{2}$ & P-value & $\begin{array}{c}\beta \\
(95 \% \mathrm{CI})\end{array}$ & $\mathbf{R}^{2}$ & P-value \\
\hline Contralateral Intact ACL Signal Intensity & $-0.66(-36.52,35.20)$ & .00 & .964 & $0.64(-0.49,1.76)$ & .30 & .204 \\
\hline Contralateral Intact ACL Cross-sectional Area & $0.42(-0.74,1.57)$ & .15 & .395 & $0.01(-0.03,0.06)$ & .11 & .457 \\
\hline *Normalized Femoral Stump Length & $-0.15(-0.74,0.44)$ & .05 & .566 & $-0.01(-0.03,0.00)$ & .46 & .045 \\
\hline *Normalized Tibial Stump Length & $0.09(-0.51,0.68)$ & .02 & .746 & $0.01(0.00,0.03)$ & .30 & .129 \\
\hline Lateral Tibial Slope & $0.11(-2.37,2.59)$ & .00 & .919 & $0.07(0.01,0.12)$ & .57 & .019 \\
\hline Medial Tibial Slope & $0.44(-1.77,2.66)$ & .03 & .651 & $0.05(0.00,0.11)$ & .39 & .075 \\
\hline Medial Tibial Depth & $-0.21(-7.07,6.66)$ & .00 & .945 & $-0.17(-0.32,-0.01)$ & .48 & .039 \\
\hline Notch Width (pre-op) & $3.51(0.39,6.64)$ & .50 & .033 & $0.05(-0.09,0.18)$ & .09 & .434 \\
\hline Notch Width (post-op) & $1.89(0.76,3.03)$ & .69 & .006 & $0.01(-0.05,0.08)$ & .03 & .639 \\
\hline Amount of Notchplasty & $3.06(1.17,4.95)$ & .68 & .007 & $0.01(-0.10,0.11)$ & .01 & .852 \\
\hline
\end{tabular}

$\mathrm{N}=9$ for all except normalized signal intensity and cross-sectional area of the contralateral intact ACL ( $\mathrm{N}=7$ ).

Normalized to total ACL length. 
Table 3:

Regression coefficients for the MRI-based predictors of the reconstructed graft cross-sectional area/signal intensity at 24 months. There were no significant associations. $\beta$ is regression slope, representing change in the outcome per unit change in the predictor.

\begin{tabular}{|c|c|c|c|c|c|c|}
\hline \multirow[b]{3}{*}{ MRI-based Predictors } & \multicolumn{6}{|c|}{ Graft Properties (24 months) } \\
\hline & \multicolumn{3}{|c|}{ Cross-Sectional Area } & \multicolumn{3}{|c|}{ Signal Intensity } \\
\hline & $\begin{array}{c}\beta \\
(95 \% \mathrm{CI})\end{array}$ & $\mathbf{R}^{2}$ & P-value & $\begin{array}{c}\beta \\
(95 \% \mathrm{CI})\end{array}$ & $\mathbf{R}^{2}$ & P-value \\
\hline Contralateral Intact ACL Signal Intensity & $-11.02(-44.45,22.42)$ & .13 & .436 & $0.16(-0.46,0.79)$ & .08 & .529 \\
\hline Contralateral Intact ACL Cross-sectional Area & $0.32(-0.50,1.14)$ & .16 & .368 & $-0.01(-0.02,0.01)$ & .28 & .224 \\
\hline Normalized Femoral Stump Length & $0.31(-1.12,1.75)$ & .06 & 600 & $0.02(0.00,0.04)$ & .53 & .062 \\
\hline Normalized Tibial Stump Length & $-0.35(-1.20,0.50)$ & .18 & .339 & $-0.01(-0.02,0.01)$ & .30 & .203 \\
\hline Lateral Tibial Slope & $-2.92(-8.06,2.23)$ & .30 & .205 & $0.04(-0.07,0.14)$ & .13 & .418 \\
\hline Medial Tibial Slope & $-2.38(-6.51,1.76)$ & .30 & .200 & $0.05(-0.03,0.12)$ & .33 & .174 \\
\hline Medial Tibial Depth & $1.27(-17.09,19.62)$ & .01 & .866 & $0.06(-0.27,0.39)$ & .04 & .668 \\
\hline Notch Width (pre-operative) & $1.09(-2.50,4.68)$ & .11 & 609 & $-0.03(-0.09,0.03)$ & .26 & .246 \\
\hline Notch Width (post-operative) & $0.13(-4.29,4.56)$ & .01 & .941 & $-0.02(-0.10,0.06)$ & .06 & .604 \\
\hline Amount of Notchplasty & $-2.23(-7.32,2.87)$ & .20 & .312 & $0.04(-0.05,0.13)$ & .20 & .311 \\
\hline
\end{tabular}

$\mathrm{N}=7$ for all analyses 